sympathetic ophthalmitis is due to an external organism, it is not right that the fear of the disease should debar the patient from the promise of benefits held out apparently only by iris-incarceration. The advantages apparently realizable are uniformly permanent and yet moderate drainage, elimination of hypotony and of the conjunctival bleb, also of the dangers of a large incision and of delayed re-formation of the anterior chamber.

It is the surgeon's business to exclude all possibility of surface infection, and there is sufficient evidence that it can be done. Unless exceptionally reliable culture tests are available, some proved means of clearing away conjunctival bacteria should always be used. The only means with which I am personallv acquainted is the maximal perchloride irrigation that has stood the test of a quarter of a century of practical experience in India. This, with swabbing of the lid margins by the same solution, causes a little conjunctival and lid swelling. Conjunctival injection is controlled during operation by adrenalin. There is a variable degree of smarting for a few hours afterwards, and there is often a little mucoid discharge later. But it never does any harm, and I have never found that the patients object to it. They infinitely prefer it to running the slightest risk.

Also the method of operating should be as subconjunctival as possible. I should like merely to mention a technique that promises particularly well. Dissatisfied with imperfect subconjunctival drainage in operating with the triangular keratome, I have lately gone back to the narrow tapering glaucoma knife. The sclerocorneal incision is made after sliding the conjunctiva. The only opening in the latter is vertical or oblique, $4-5 \mathrm{~mm}$. long, in the upper outer quadrant of the eye, $4-5 \mathrm{~mm}$. away from the cornea. And the subconjunctival drainage up to date appears perfect. But I have only done a few of these operations, and it is too early to speak of the final results.

\title{
AN INTERESTING EXAMPLE OF HEREDITARY DISLOCATION OF THE LENS OCCURRING IN FOUR SUCCESSIVE GENERATIONS
}

BY

Elizabeth Pace Cameron, M.B., Ch.B., D.P.H. CARDIFF

THE following example of hereditary dislocation of the lens occurring in four successive generations, appears to be of sufficient interest to be worth recording. There have been fourteen persons affected of whom only one was a male. Five have had to be trained in institutions for the blind. In every case, both lenses have been 
dislocated, the vision has been seriously affected and a squint, either convergent or divergent, has been apparent.

The family was brought to my notice when three sisters who attended my school refraction clinic were found to be suffering from subluxation of both lenses, the mother who accompanied them having the same defect. Their condition was briefly as follows :

(1) L.J. (female), aged 13 years, had vision R. $1 / 60$; L. 1/60. Both lenses were partially dislocated upwards so that their crescentic edges lay across the centre of the pupils. Vision with +12.00 D. sph. was R. 3/60: L. 3/60. No abnormality was detected in the fundus. The case was certified for a special school.
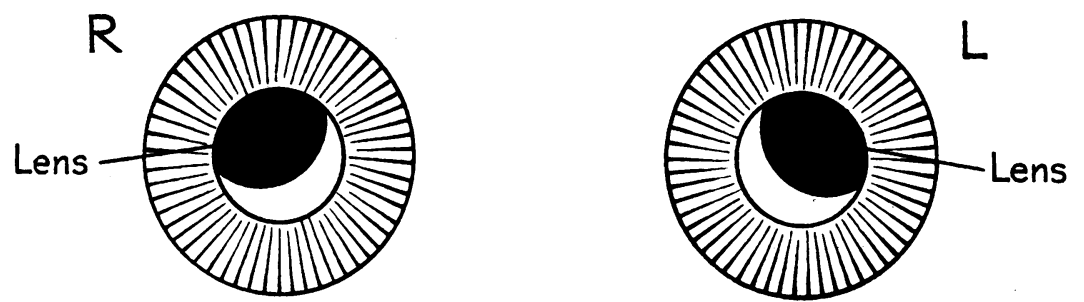

(2) E.C. (female), aged 11 years, had vision R. $3 / 60$; L. 3/60 which was not improved by lenses. The dislocation was similar to the above and the fundi appeared normal. She also was sent to a school for the blind.

(3) H.V. (female), aged 6 years, could not read letters but her vision seemed to be $R$. fingers counted at 8 inches distance; L. 1/60. The right lens was much displaced to the temporal side leaving most of the pupil free, and therefore vision was improved by a +11.00D. sph., and it was hoped that by wearing this lens the child would develop considerably the function of the retina. In the left eye, the lower margin of the lens was across the centre of the pupil. The fundi were both normal.
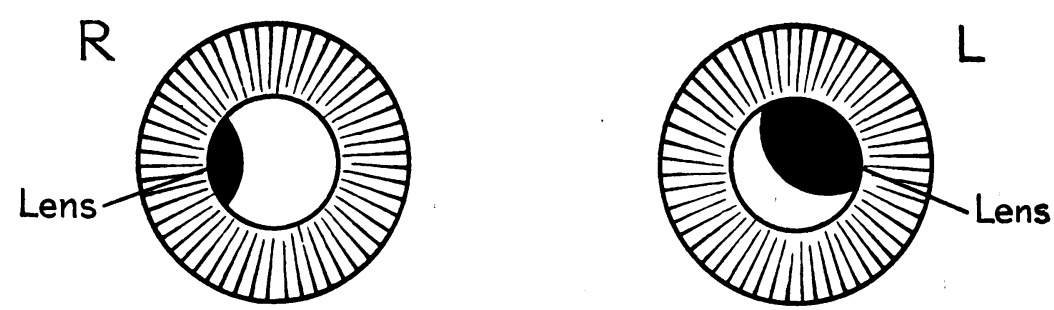

(4) Mrs. G. had dislocated lenses but as her pin-point pupils did not dilate under atropin it was impossible to make out the details. She had tremulous irides and vision R. $3 / 60 ; \mathrm{L} .3 / 60$ which was improved by $+11.00 \mathrm{D}$. sph. to R. $5 / 60 ;$ L.5/60. 
The family history was found to be interesting. The condition can be traced to the great-grandmother who was the only one of seven children to be affected. Her only child, the grandmother of my patients, inherited the defect and of her six children four are affected including Mrs. G. Mrs. G. has five children, the three reported above and two unaffected (one boy and one girl). Mrs. G.'s elder sister, Anna Jane, was trained in a "blind" institution, and of her four children, one girl and one boy are normal, while one girl and one boy are affected, and have been educated as "blind" (this is the only boy in the series). Mrs. G.'s brother, Tom, and sister, May, are unaffected, Tom's two children and May's four children being normal.

Sophia, a younger sister of Mrs. G., has dislocated lenses, but sees moderately well with strong convex glasses; her two boys by a first husband are normal, while a daughter by a second husband is affected; her baby boy has not shown signs of the defect. Sarah, the youngest of Mrs. G.'s sisters has inherited the condition, and of her three children, the two girls are victims while the boy has escaped.

The following diagrams illustrate the position:

* Means affected with subluxation of both lenses.

* Great-grandmother

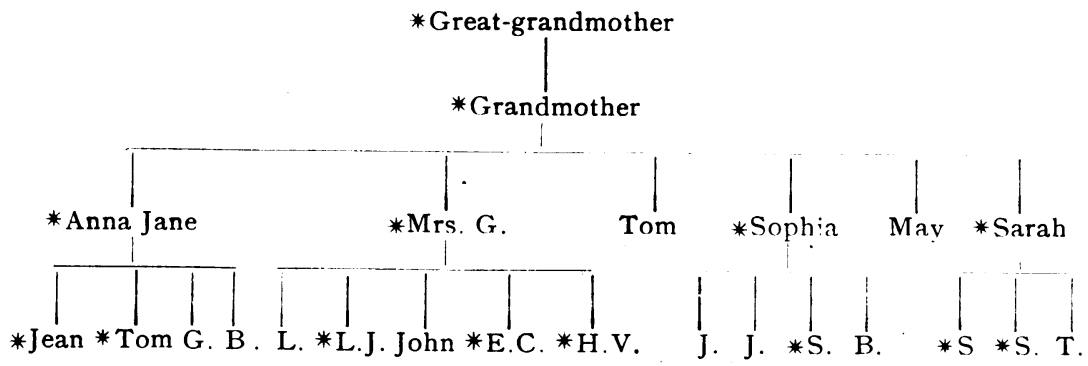

$\because$ Affected female

t Affected male

\& Unaffected female

$\delta$ Unaffected male

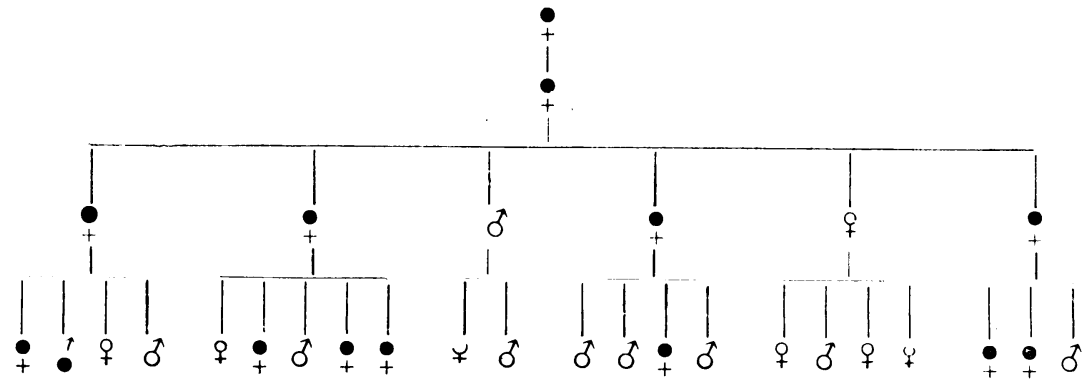

I am indebted to Mr. D. Leighton Davies, M.D., F.R.C.S., for seeing some of these cases and suggesting that they should be published. 